\title{
A rapid and sensitive assay of intercellular coupling by voltage imaging of gap junction networks
}

\author{
Federico Ceriani ${ }^{1,2}$ and Fabio Mammano $0^{1,2,3^{*}}$
}

\begin{abstract}
Background: A variety of mechanisms that govern connexin channel gating and permeability regulate coupling in gap junction networks. Mutations in connexin genes have been linked to several pathologies, including cardiovascular anomalies, peripheral neuropathy, skin disorders, cataracts and deafness. Gap junction coupling and its patho-physiological alterations are commonly assayed by microinjection experiments with fluorescent tracers, which typically require several minutes to allow dye transfer to a limited number of cells. Comparable or longer time intervals are required by fluorescence recovery after photobleaching experiments. Paired electrophysiological recordings have excellent time resolution but provide extremely limited spatial information regarding network connectivity.
\end{abstract}

Results: Here, we developed a rapid and sensitive method to assay gap junction communication using a combination of single cell electrophysiology, large-scale optical recordings and a digital phase-sensitive detector to extract signals with a known frequency from Vf2.1.Cl, a novel fluorescent sensor of plasma membrane potential. Tests performed in HeLa cell cultures confirmed that suitably encoded Vf2.1.Cl signals remained confined within the network of cells visibly interconnected by fluorescently tagged gap junction channels. We used this method to visualize instantly intercellular connectivity over the whole field of view (hundreds of cells) in cochlear organotypic cultures from postnatal mice. A simple resistive network model reproduced accurately the spatial dependence of the electrical signals throughout the cellular network. Our data suggest that each pair of cochlear non-sensory cells of the lesser epithelial ridge is coupled by 1500 gap junction channels, on average. Junctional conductance was reduced by $14 \%$ in cochlear cultures harboring the T5M mutation of connexin30, which induces a moderate hearing loss in connexin $30^{\text {T5M/T5M }}$ knock-in mice, and by $91 \%$ in cultures from connexin $30^{-1-}$ mice, which are profoundly deaf.

Conclusions: Our methodology allows greater sensitivity (defined as the minimum magnitude of input signal required to produce a specified output signal having a specified signal-to-noise ratio) and better time resolution compared to classical tracer-based techniques. It permitted us to dynamically visualize intercellular connectivity down to the 10th order in non-sensory cell networks of the developing cochlea. We believe that our approach is of general interest and can be seamlessly extended to a variety of biological systems, as well as to other connexin-related disease conditions.

Keywords: Connexins, Electrical coupling, Dye coupling, Genetic deafness, Voltage sensitive dye, Digital phase-sensitive detector

\footnotetext{
* Correspondence: fabio.mammano@unipd.it

1Dipartimento di Fisica e Astronomia "G. Galilei", Università di Padova,

Padova 35131, Italy

${ }^{2}$ Istituto Veneto di Medicina Molecolare, Fondazione per la Ricerca Biomedica

Avanzata, Via G. Orus, 2, Padova 35129, Italy

Full list of author information is available at the end of the article
} 


\section{Background}

Cell-cell communication mediated by gap junctions is crucial to a variety of cellular functions, including the regulation of cell growth, differentiation and development [1]. In electrically excitable cells, gap junctions provide low-resistance pathways, traditionally referred to as electrical synapses, and permit transmission of electrical signals between adjacent cells. In the brain, electrical synapses have been shown to be important for enabling and detecting neuronal synchrony $[2,3]$ and to regulate lineage-dependent microcircuit assembly [4]. In the heart, the ability to synchronize groups of cells is crucial to achieve a coordinated mechanical output $[5,6]$. In non-excitable cells, gap junctions permit to share metabolic demands across groups of cells, enable the exchange of signaling molecules $[7,8]$ and the spatial buffering of potassium ions [9].

Virtually all cells in solid tissues are coupled by gap junctions [1], thus it is not surprising that mutations in connexin genes have been linked to a variety of human diseases, including cardiovascular anomalies, peripheral neuropathy, skin disorders, cataracts and deafness [10-12]. Gap junction channels in the mammalian cochlea, the site of the sense of hearing, are formed primarily by connexin 26 and connexin 30 proteins encoded by nonsyndromic hearing loss and deafness (DNFB1) genes GJB2 and GJB6, respectively [13]. Cochlear connexins are expressed very early on in development and interconnect virtually all types of non-sensory cells [14-16]. Morphological analysis of cochleae from different strains of mice with (targeted) ablation of connexin 26 or connexin30 provide evidence of incomplete or arrested development ensuing in defects of hearing acquisition [12].

The most widely used approach to monitor intercellular communication employs optical methods to track the movement of tracer molecules between neighboring cells. However, the sensitivity of this technique depends on the junctional permeability of the tracer employed, which varies significantly with the size of the permeant molecule and the type of gap junction channels. Sensitivity can be increased by prolonging the loading time or by employing smaller tracer molecules (e.g. serotonin [17]).

Here, we used cochlear organotypic cultures to unravel the potential of $\mathrm{Vf2}$.1.Cl, a member of the novel VoltageFluor (VF) family of fluorescent sensors [18]. VF dyes detect voltage changes by modulation of photoinduced electron transfer (PeT) from an electron donor through a synthetic molecular wire to a fluorophore. They have large, linear, turn-on fluorescence responses to depolarizing steps (20-27\% fluorescence change per $100 \mathrm{mV})$, fast kinetics $(\tau<<140 \mu \mathrm{s})$ and negligible capacitative loading. We exploited the $\mathrm{Vf} 2.1$.Cl voltage sensitive dye [18] to probe dynamically the extent of gap junction coupling by a combination of single cell electrophysiology, large scale optical recordings and a digital phase-sensitive detector of fluorescence signals. Our method is readily applicable to a variety of cellular systems, as it requires only a patch-clamp amplifier to inject sinusoidal electrical signals at fixed frequency and amplitude in a single cell and a fluorescence microscope to track optically the VF dye response at the frequency of the stimulus throughout the network.

\section{Results}

\section{In-situ calibration of the Vf2.1.Cl voltage sensitive dye}

Organotypic cultures of cochlear explants from postnatal mice permit to investigate the patho-physiology of gapjunction-mediated intercellular signaling in a readily accessible whole-organ context [19-24]. In order to calibrate the voltage response of the fluorescent sensor in our experimental conditions (see Methods), we loaded organotypic cultures from wild type mice, euthanized at postnatal day 5 (P5), with Vf2.1.Cl. We then performed paired whole-cell patch clamp recordings from cochlear non-sensory cells of the lesser epithelial ridge. We stepped the voltage $V_{0}$ of the patch clamp amplifier connected to one cell (cell 1, Figure 1A) in $10 \mathrm{mV}$ increments (Figure 1B, black trace) from the zero current potential $(-61 \pm 2 \mathrm{mV}, n=15$ cells) while monitoring the membrane potential $\left(V_{m}\right)$ (Figure $1 \mathrm{~B}$, red trace) of a nearby cell (cell 2, Figure 1A) maintained under current-clamp conditions with a second amplifier. At the same time, we measured $\mathrm{Vf} 2.1$.Cl fluorescence emission $(F)$ from cell 2 (Figure 1B, blue trace). Data in Figure $1 \mathrm{~B}, \mathrm{C}$ highlight a linear relationship between the change in membrane potential $\left(\Delta V_{m}\right)$ and the corresponding fractional change $\left(\Delta F / F_{0}\right)$ in $\mathrm{Vf2}$.1.Cl fluorescence emission. Note that both $\Delta F / F_{0}$ and $\Delta V_{m}$ were detected from cell 2. The correlation coefficient between $\Delta F / F_{0}$ and $\Delta V_{m}$ was $R=0.98$ ( $n=5$ paired recordings in 3 cultures) and a linear fit to the data (Figure 1C, solid line) yielded a responsivity (slope) $m=0.23 \pm 0.03 \Delta F / F_{0} /$ $\mathrm{mV}$ (i.e. $23 \pm 3 \%$ per $100 \mathrm{mV}$ ). Both $\Delta V_{m}$ and $\Delta F / F_{0}$ responses were suppressed after incubating the culture for 20 minutes in $100 \mu \mathrm{M}$ carbenoxolone (CBX, Figure 1D), a non-selective blocker of gap junction channels [25].

Based on this calibration, we estimated optically the voltage step in cell $1\left(\Delta V_{1}\right)$ corresponding to a given voltage command $\Delta V_{0}$ delivered by the patch clamp amplifier. On average, $\Delta V_{0}=70 \mathrm{mV}$ yielded a $\Delta V_{1}=$ $22 \pm 4 \mathrm{mV}(n=5)$ in wild type cultures. We then derived the access resistance of the patch pipette connected to cell 1 as $R_{a}=\Delta\left(V_{0}-V_{1}\right) / \Delta I$, where $\Delta I=6.8 \pm 1.1 \mathrm{nA}$ $(n=5)$ is the current step measured by the amplifier. The value we obtained, $R_{a}=7.8 \pm 0.9 \mathrm{M} \Omega(n=5)$, is in excellent agreement with the estimate provided by the membrane test of the patch clamp software, $R_{a(\text { patch })}=$ $7.5 \pm 1.2 \mathrm{M} \Omega(n=5)$. 


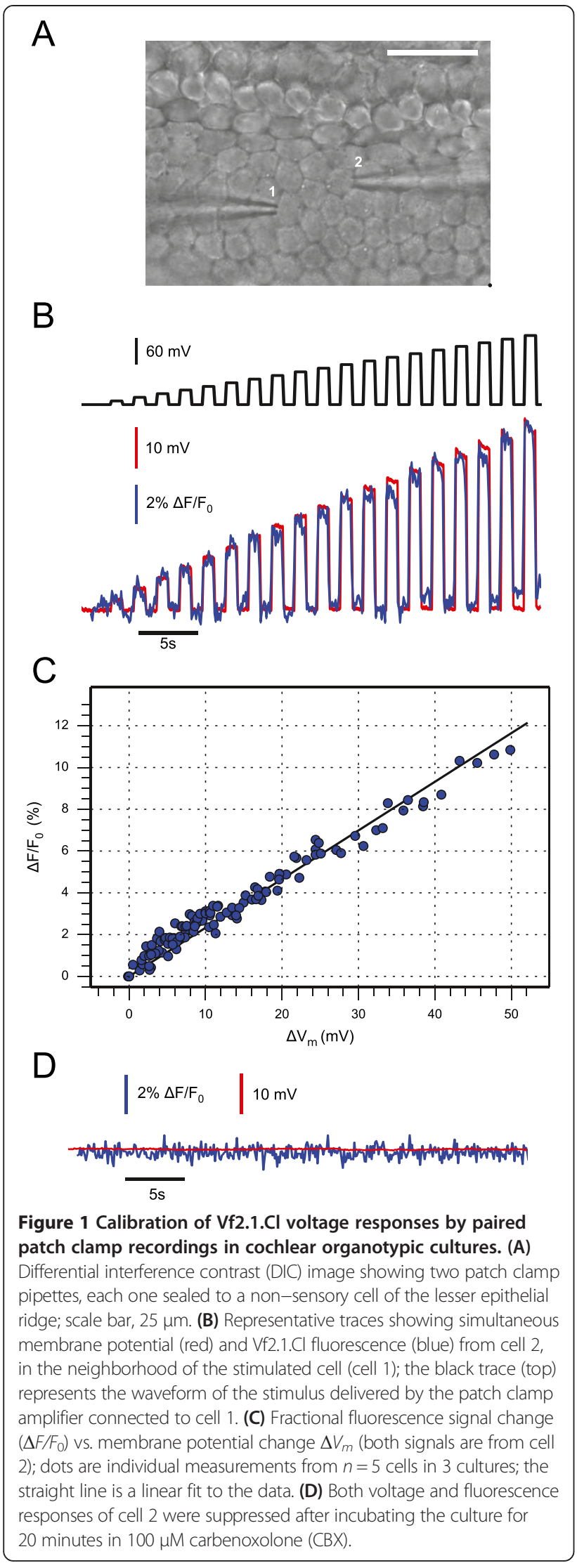

A digital phase-sensitive detector of Vf2.1.Cl signals visualizes and quantifies network connectivity

Paired electrophysiological recordings, such as those in Figure 1, have excellent time resolution but provide extremely limited spatial information regarding network connectivity. The main goal of the present study was to visualize rapidly network connectivity using large-scale optical recordings of $\mathrm{Vf2}$.1.Cl florescence in different preparations and experimental conditions. The calibration procedure reported in Figure 1 yielded maximal fluorescence changes $\Delta F / F_{0}$ in cell 2 , close to cell 1 , which rarely exceeded $10 \%$. Electrical signals spreading passively through a resistive network are expected to attenuate rapidly with distance from the source (i.e. cell 1) and fluctuations due to photon shot noise hamper their detection [26]. We sought to overcome these limitations by the following procedure.

We loaded cochlear organotypic cultures from P5 mice with the Vf2.1.Cl dye and delivered a sinusoidal voltage command, also named carrier wave (frequency $v=0.5 \mathrm{~Hz}$, amplitude $35 \mathrm{mV}$ ) to the patch clamp amplifier connected to one cell of the network (cell 1, Figure 2A). In wild type cultures, this stimulation elicited instantly sinusoidal optical signals of $\mathrm{Vf} 2.1 \mathrm{Cl}$ fluorescence at the frequency $v$ of the carrier wave (reference frequency) in virtually all cells of the network within the field of view (Additional file 1: Movie S1). We then used the off-line digital phase-sensitive detector (also known as lock-in amplifier) described in the Methods to extract Vf2.1.Cl signal amplitude $A(x, y)$ at each network location $(x, y)$ at the reference frequency (Figure 2B). This method works because noise at frequencies other than $v$ is rejected and does not affect the measurement [27]. Throughout this article, relative amplitude refers to $A(x, y) / A_{1}$ where $A_{1}$ is signal amplitude at the reference frequency in the stimulated cell. At each point $(x, y)$, relative amplitude values remained stable for tens of seconds during carrier wave delivery to cell 1 , but decreased rapidly with distance from this cell (Figure 2C and D). At the single pixel level, the standard deviation $\sigma$ of the signal $A(x, y)$ returned by the digital phase-sensitive detector scaled correctly as the square root of the number $N$ of integration cycles (Figure 2E). Note that $\sigma \approx 2.2 \mathrm{mV}$ at $N=1$ and $\sigma \approx 0.5 \mathrm{mV}$ at $N=25$; reaching sub-mV sensitivity required $N \geq 5$.

To estimate cell network extension, we computed $A(x, y)$ by integrating Vf2.1.Cl signals over $N=5$ carrier wave cycles (Figure 3). This approach permitted us to discriminate rapidly (10 s per recording) network connectivity of wild type cultures (Figure 3A, top left) from that of genetically modified connexin $30^{\mathrm{T} 5 \mathrm{M} / \mathrm{T} 5 \mathrm{M}}$ (top right) and connexin $30^{-/-}$ (bottom left) cultures [28]. Incubating wild type cultures for 20 minutes in $100 \mu \mathrm{M} \mathrm{CBX}$ confined the $\mathrm{Vf} 2.1$.Cl signal to the stimulated cell (bottom right), indicative of junctional conductance $\left(g_{j}\right)$ collapse over the entire network. 


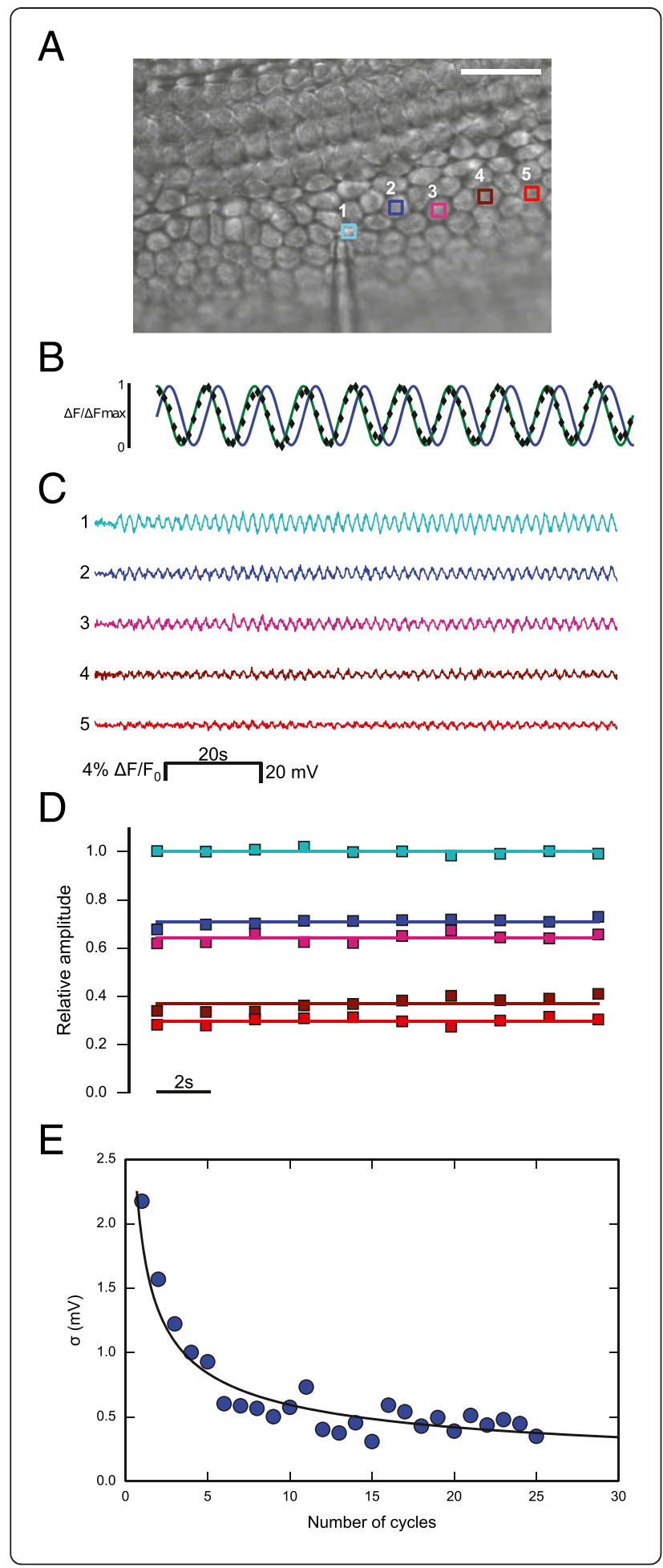

Figure 2 Illustrating phase-sensitive detection of Vf2.1.Cl fluorescence responses. (A) DIC image showing a single patch clamp pipette sealed to a non-sensory cell of the lesser epithelial ridge (cell 1, zero current potential $-66 \mathrm{mV}$ ); scale bar, $25 \mu \mathrm{m}$. (B) Black diamonds: normalized optical signals from a specific cell network location; green trace: unit amplitude carrier wave delivered to cell 1; blue trace: its phase-shifted counterpart used in the computation of signal amplitude (see Methods). (C) Calibrated optical responses from the five regions of interest (ROIs) shown in (A) during a typical stimulation protocol. A low order polynomial fit was subtracted to the raw traces to compensate for the effects of photobleaching (see Methods). (D) Relative amplitude signals derived by integrating traces shown in $(\mathbf{C})$ over a single carrier wave cycle $(N=1)$. (E) The standard deviation $\sigma$ of the single pixel amplitude signal $A(x, y)$ is plotted against the number $N$ of integration cycles (see Methods); the black solid line is a least square fit to the data with the function $\sigma_{1} / N^{1 / 2}$ where $\sigma_{1}=1.9 \mathrm{mV}$.

For statistical comparison, we increased the precision of these steady-state measurements by integrating $\mathrm{Vf} 2.1 . \mathrm{Cl}$ signals over $N=25$ carrier wave cycles (50 s per recording) and measured the culture area where $A(x, y)$ exceeded an arbitrary threshold value corresponding to $2 \sigma \approx 1.0 \mathrm{mV}$ (suprathreshold area; pooled results are summarized in Figure 3B). Compared to wild type cultures, suprathreshold areas in connexin $30^{\mathrm{T} 5 \mathrm{M} / \mathrm{T} 5 \mathrm{M}}$ and connexin $30^{-/-}$cultures were significantly shifted towards lower values $(p=0.03$ and $p=0.006$, respectively; Mann-Whitney $U$ test; $n=5$ cultures for each genotype). In wild type cultures, the lower quartile, the median, and upper quartile of suprathreshold area values were respectively: $17230,18430,18970 \mu^{2}$; the corresponding values in connexin $30^{\mathrm{T} 5 \mathrm{M} / \mathrm{T} 5 \mathrm{M}}$ cultures were: 10730, 13550, $15100 \mathrm{\mu m}^{2}$; finally, in connexin $30^{-/-}$cultures, they were: $2730,3550,5300 \mu^{2}$.

\section{A simple resistive network model accounts for the spatial dependence of $\mathrm{Vf2}$.1.Cl signals}

To gain further insight into the spatial dependence of the data shown in Figure 3, we modeled the cell network as a collection of nodes (individual non-sensory cells) forming an hexagonal mesh that reflects the anatomy [29]. In this model, nodes were coupled by resistive links with identical junctional conductance $g_{j}$. Each node was also connected to ground by a resistor with conductance $g_{m}$ representing cell membrane (Figure 4). We pooled data from $n=5$ cultures for each genotype at equal distances from the stimulated cell along the coiling axis of the cochlea and plotted the result versus this distance. Finally, we obtained leastsquare fits to these averaged data using the network model with $g_{j}$ as the only free parameter. The results were: $g_{j}=$ $206 \mathrm{nS}$ for wild type, $177 \mathrm{nS}$ for connexin $30^{\mathrm{T} 5 \mathrm{M} / \mathrm{T} 5 \mathrm{M}}$ and $19 \mathrm{nS}$ for connexin $30^{-/-}$cultures.

\section{Application to network dynamics}

In patho-physiological conditions, gap junction networks are dynamically regulated by a variety of mechanisms that 

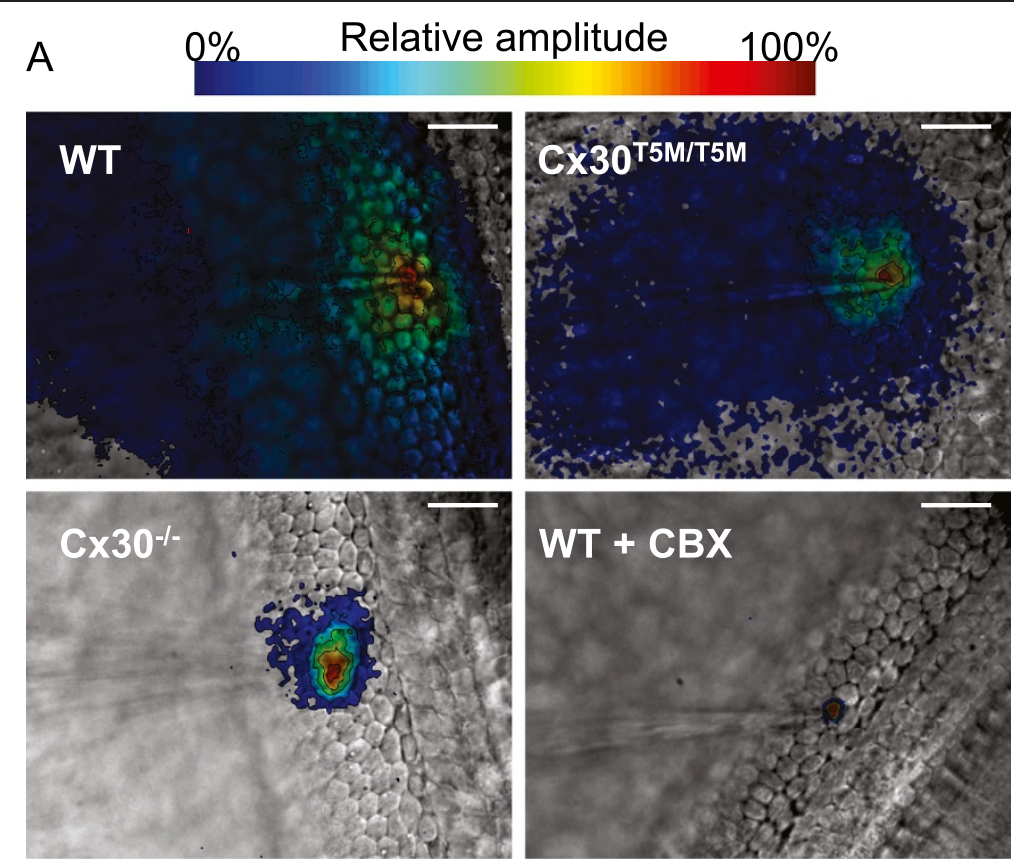

B

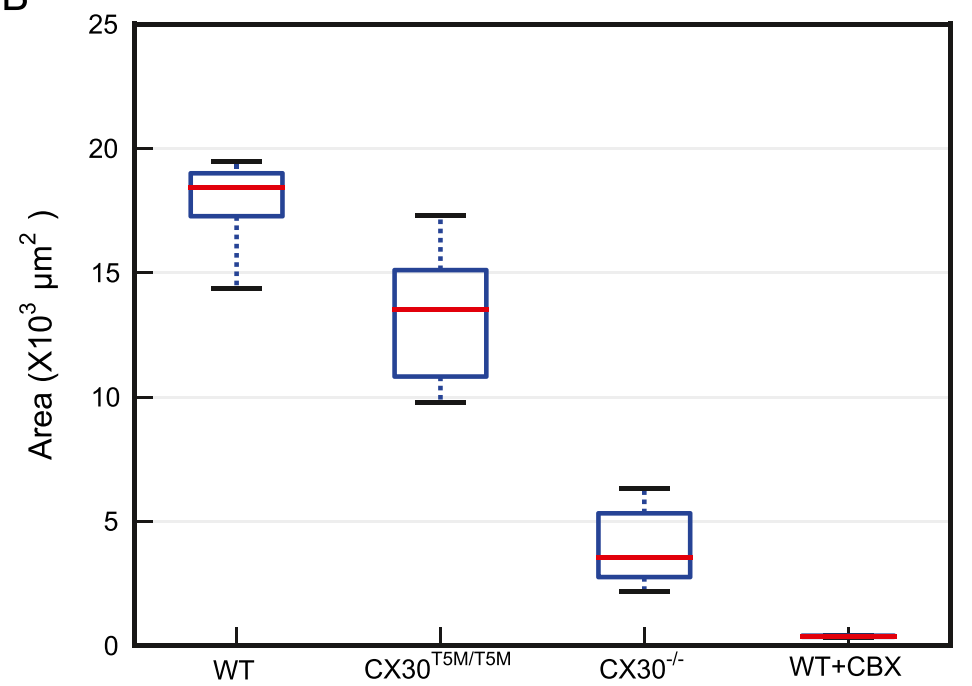

Figure 3 Direct readout of network connectivity by large-scale optical recordings of $\mathrm{Vf2}$.1.Cl fluorescence responses to a $0.5 \mathrm{~Hz} 35 \mathrm{mV}$ carrier wave. (A) Representative false-color images showing the spatial distribution of Vf2.1.Cl relative amplitude signals in cultures from P5 wild type (top left), connexin30 ${ }^{\mathrm{TSM} / T 5 \mathrm{M}}$ (top right) and connexin30-/- (bottom left) mice; the bottom right image refers to a wild type culture in which gap junction channels were blocked by 20 min incubation with CBX $(100 \mu \mathrm{M})$; in this image, the area with a residual relative amplitude signal $\left(226 \mu \mathrm{m}^{2}\right)$ is very close to the average area of a single cell in this part of the culture ( $210 \pm 7 \mu \mathrm{m}^{2}, n=10$ cells); scale bars, $25 \mu \mathrm{m}$. (B) Suprathreshold area distributions shown in box plot form; see main text for details.

govern connexin channel permeability and gating $[1,7,8,20,30]$. Our next goal was to track dynamical changes in cell network connectivity by applying a digital phase-sensitive detector to Vf2.1.Cl signals. For this series of recordings, we limited time integration to $N=4$ carrier wave cycles while transiently superfusing cochlear cultures with an extracellular medium saturated with $100 \% \mathrm{CO}_{2}$ to produce carbonic acid
$\left(\mathrm{H}_{2} \mathrm{CO}_{3}\right)$. In its non-dissociated form $\mathrm{H}_{2} \mathrm{CO}_{3}$ is membrane permeable and causes a rapid and reversible closure of gap junction channels $[7,30]$. This manipulation led to a reduction in the number of cells coupled to the stimulated cell, accompanied by a transient increase in $\mathrm{Vf2}$.1.Cl fluorescence in the neighborhood of this cell (Figure 5A, B and Additional file 2: Movie S2). To mimic the time course of the events shown in Figure $5 \mathrm{~A}$, B, we 


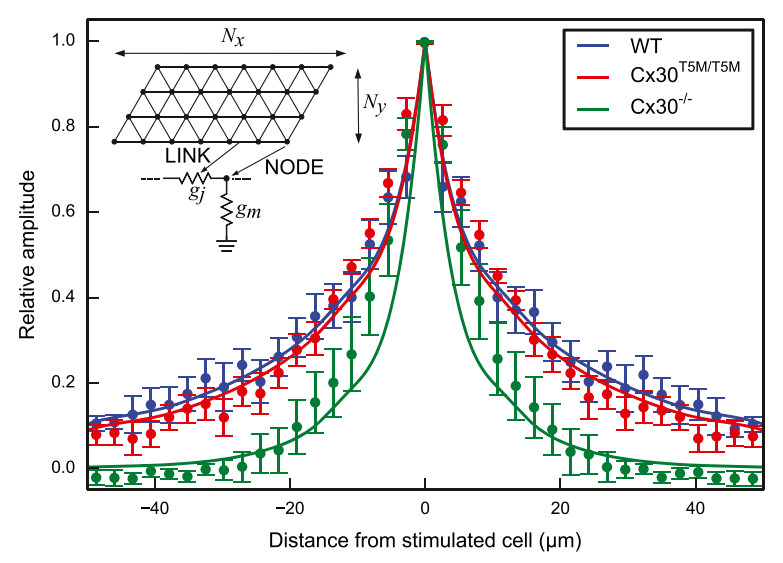

Figure 4 Data fit by a simple resistive network model that reflects the anatomy. The inset shows model scheme. Simulations were performed using the ngspice software (http://ngspice. sourceforge.net). $N_{x}=45$ and $N_{y}=15$ indicate the number of rows and columns in the grid, respectively. Each node represents one cell and each link represents a resistive connection between adjacent nodes. The patch pipette connected to cell 1 was simulated as a variable voltage source connected to one node of the grid through an access resistance $R_{a}=7.8 \mathrm{M} \Omega$ (not shown). A single value for membrane conductance $\left(g_{m}=8.3 \mathrm{nS}\right)$ and junctional conductance $\left(g_{j}\right)$ were used throughout the network. $g_{j}$ was left as the only free parameter in the simulations and its value was derived using a maximum-likelihood algorithm.

simply assumed that the $g_{j}$ of the network model represented in Figure 4 undergoes a time-dependent exponential decrease from $206 \mathrm{nS}$ to $2 \mathrm{nS}$ with a time constant of $7 \mathrm{~s}$ (Figure 5C).

\section{Application to immortalized cell lines}

A number of gap junction communication studies are performed in expression systems and/or immortalized cell lines. To demonstrate the applicability of the method highlighted above to this important area of research, we used a clone of HeLa cells virtually devoid of connexins (see Methods) that were either left untreated (HeLa parental) or transiently transfected with a construct expressing human connexin26 fused in tandem with a cyan fluorescent protein (CFP) reporter (hCx26CFP) [20]. These chimerical proteins localized to the plasma membrane at regions of contact between adjacent cells and also formed distinct fluorescent puncta in the cytoplasm, as previously described $[20,31,32]$. Confluent HeLa cell cultures were loaded with the Vf2.1.Cl dye and subjected to the same patch-clamp protocol used in organotypic cochlear cultures. In HeLa parental cultures, the Vf2.1.Cl signal remained confined to the stimulated cell (Figure 6A). In transfected cultures, the Vf2.1.Cl signal displayed variable degrees of cell-to-cell spreading, reflecting the number of transfected cells connected to the stimulated cell by hCx26-CFP gap junction channels (Figure 6B, C and D).

\section{Discussion}

We report here the application of the novel $\mathrm{Vf} 2.1 \mathrm{Cl}$ fluorescent sensor [18] to voltage imaging in cell networks coupled by gap junction channels. We focused our attention on non-sensory cell networks of the developing cochlea and used organotypic cultures from P5 mice as model system. Our in situ calibration yielded an estimated responsivity of $23 \pm 3 \% \Delta F / F_{0}$ per $100 \mathrm{mV}$ change of the cell membrane potential $V_{m}$ (Figure 1), in substantial agreement with measurements performed in HEK293 cells (26\% per $100 \mathrm{mV})$ [18]. The limited dispersion of the data in Figure $1 \mathrm{C}$, which were acquired under different $F_{0}$ conditions, indicates that the responsivity is fairly independent of the loading conditions. However, $F_{0}$ does affect the signal-to-noise ratio, which is ultimately dictated by fluctuations in the number of collected photons (see, e.g. Ref. [26]), and consequently also the sensitivity of the measurement.

The Vf2.1.Cl signal tracks the membrane potential with no detectable delay [18], a highly desirable feature that distinguishes this dye from the substantially slower voltage sensors based on fluorescent proteins. The use of these proteins is also hindered by the necessity of transfecting/ transducing target cells with a suitable expression vector [33]. In contrast, Vf2.1.Cl loads readily (15 $\mathrm{min})$ and our use of a digital phase-sensitive detector (see Methods and Figure 2) allowed us to rapidly map cellular connectivity over vast network areas (Figures 3, 4, 5 and 6). With our methodology, the time required for data collection is a multiple of the carrier wave period ( $2 \mathrm{~s}$ in our conditions). Increasing the integration interval slows down the acquisition (i.e. it reduces the temporal resolution of dynamical measurements such as those presented in Figure 5) but reduces fluctuations (Figure 2E) and thus increases both sensitivity and precision (defined as the degree to which repeated measurements under unchanged conditions show the same result).

By integrating the Vf2.1.Cl signal over $N=5$ carrier wave cycles $(10 \mathrm{~s})$, we detected intercellular connectivity down to (at least) $10^{\text {th }}$ order cells (i.e. to cells that were separated from the stimulated cells by a linear sequence of 10 adjacent neighbors), in wild type cultures. For comparison, microinjection experiments with fluorescent tracers that permeate cochlear gap junction channels (e.g. calcein, a relatively large permeant tracer that barely fits into the pore of connexin 26 channels [34]) require typically $4 \mathrm{mi}-$ nutes to allow dye transfer to $3^{\text {rd }}$ or $4^{\text {th }}$ order cells (e.g., see Supplementary Figure 6 of ref. [22]; an example of a microinjection experiment performed in this preparation with the widely used fluorescent tracer Lucifer Yellow is shown in Additional file 3: Movie S3). Comparable time 

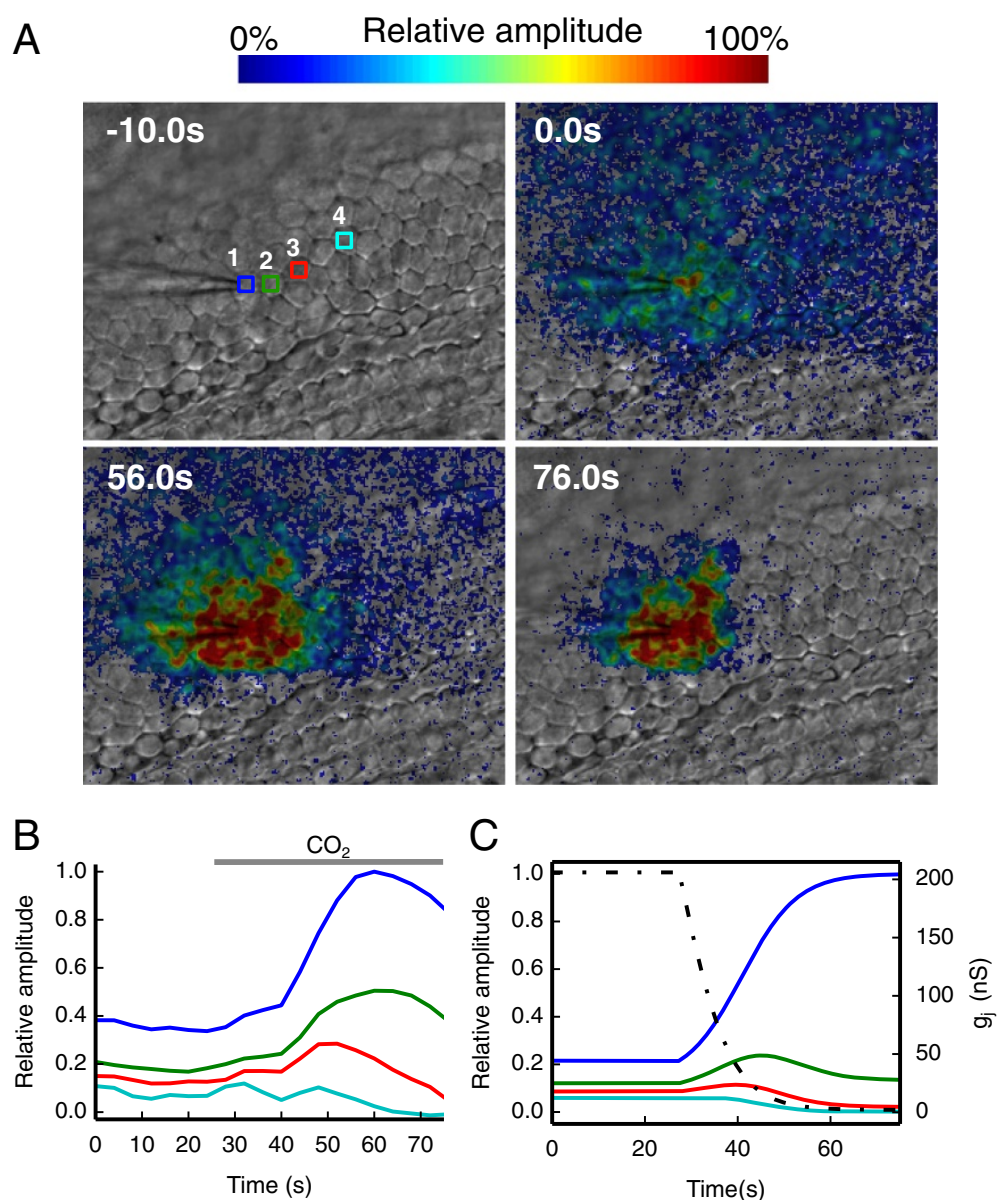

Figure 5 Effect of cell uncoupling by $\mathrm{CO}_{2}$. (A) Four selected frames from the same image sequence showing the progressive reduction of the number of cells coupled to the stimulated cell before and during exposure to $100 \% \mathrm{CO}_{2}$; the top left image was captured $10.0 \mathrm{~s}$ before the delivery of the carrier wave stimulus to cell 1; $\mathrm{CO}_{2}$ delivery started at $25 \mathrm{~s}$ and was maintained thereafter; scale bar, $25 \mu \mathrm{m}$. (B) Time course of pixel averages from the color-coded ROls shown in (A). (C) Numerical simulation of the electrical uncoupling process; the effect was mimicked by rapidly decreasing the value of junctional conductance $g_{j}$ (black dashed line) in the network model of Figure 4 from an initial value of 206 nS to 2 nS.

intervals are necessary to assay gap junction communication by fluorescence recovery after photobleaching (gap-FRAP) [35] (see, e.g. Figure 5 of ref. [36]).

The exact stoichiometry of cochlear gap junction channels in terms of connexin 26 and connexin 30 subunits is not known. Single channel currents from HeLa cells overexpressing either connexin 26 or connexin 30 homomeric channels yielded respectively values of 115 $\mathrm{pS}$ and $160 \mathrm{pS}$ for the unitary conductance $\gamma[20,32]$. A study in HeLa cells co-transfected with the cDNA of both proteins, and thus presumably forming heteromeric/ heterotypic channels, reported $\gamma$ values in a comparable range of 110-150 pS [37]. The junctional conductance $g_{j}=206 \mathrm{nS}$ we obtained by fitting wild type culture data in Figure 3 with the resistive network model of Figure 4 suggests that cochlear non-sensory cells are already well coupled at P5, by as many as $N_{\text {open }}=g_{j} / \gamma=1300$ to 1800 open channels per cell pair. An older study in isolated pairs of supporting cells of the adult guinea pig organ of
Corti reported that junctional conductance may exceed non-junctional conductance by three orders of magnitude and, at least in some cell pairs, $g_{j}$ was as large as $1 \mu \mathrm{S}$ [38] corresponding to $N_{\text {open }} \sim 10^{4}$. We are not aware of structural investigations performed in the developing cochlea. However, Forge et al. [39] noted that gap junction plaques in the supporting cells of the mature cochlea are "enormous" and they often occupy a major fraction of the plasma membrane between two adjacent cells (from 25\% to almost $100 \%$ in pillar cells). From their freeze fracture studies, Forge et al. concluded that plaques containing about $10^{4}$ channels are not rare and some may even contain $10^{5}$ channels, such as those coupling inner pillar cells in the longitudinal direction. Thus our $g_{j}$ estimate is not in contrast with the proposal that only about $10 \%$ of channels within a plaque are in the open state [40-42].

Data in Figure 3 show a $27 \%$ and $80 \%$ reduction in the median suprathreshold area respectively for connexin $30^{\mathrm{T} 5 \mathrm{M} / \mathrm{T} 5 \mathrm{M}}$ and connexin $30^{-/-}$cultures relative to 


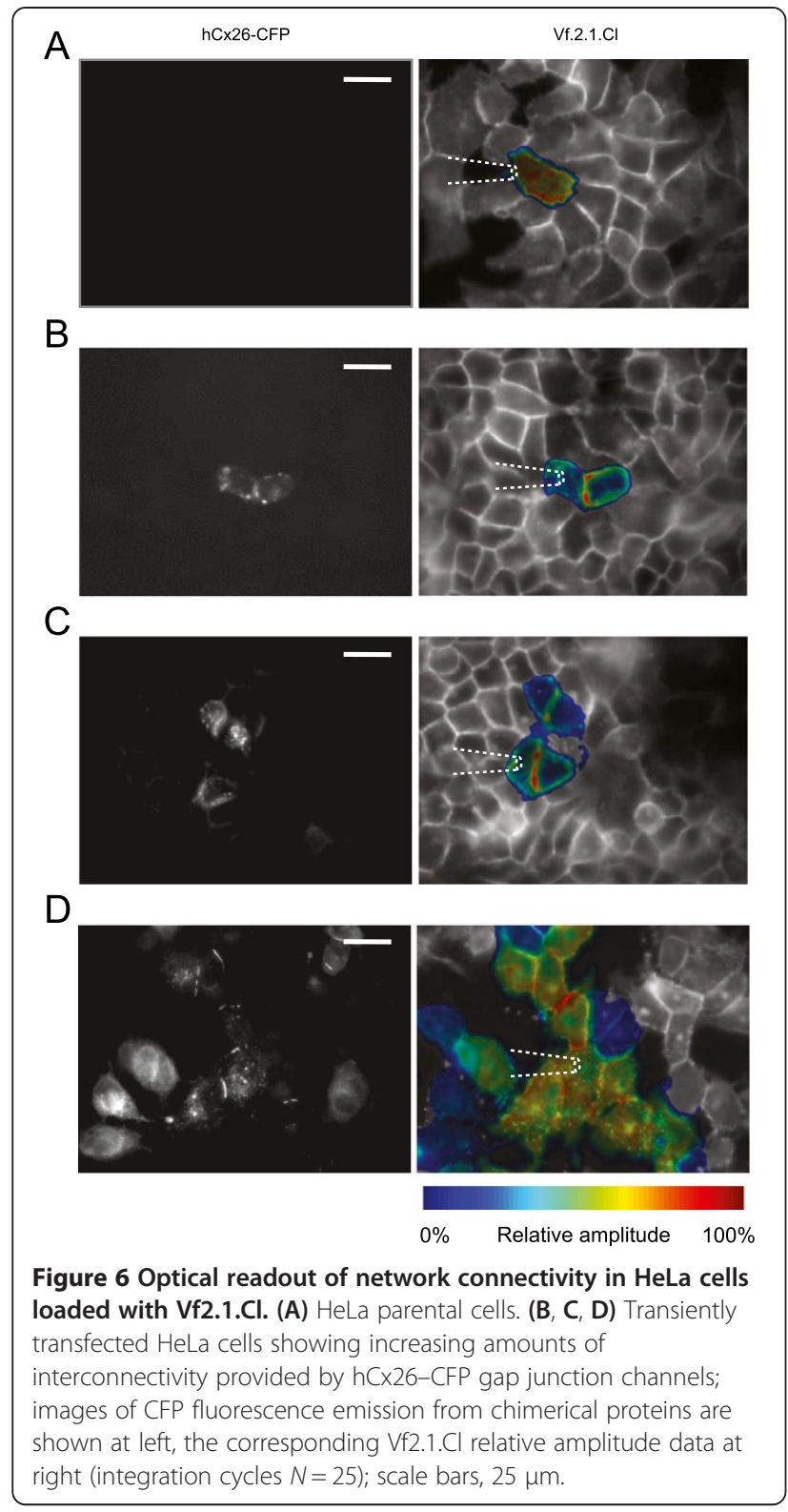

wild type cultures. Our resistive network model suggests that these reduced areas correspond to a $g_{j}$ decrease of $14 \%$ and $91 \%$ for connexin $30^{\mathrm{T} 5 \mathrm{M} / \mathrm{T} 5 \mathrm{M}}$ and connexin $30^{-/-}$ cultures, respectively. We previously reported massive down-regulation of connexin26 in the developing organ of Corti of connexin $30^{-/-}$mice [36]. Connexin26 is similarly reduced, to $10 \%$ of the wild type level, in the cochlea of adult connexin $30^{-1-}$ mice. These findings complement and extend our prior work [28], which highlighted a significant reduction in the level of dye coupling in connexin $30^{\mathrm{T} 5 \mathrm{M} / \mathrm{T} 5 \mathrm{M}} \mathrm{cul}-$ tures, whereas dye coupling was absent in connexin $30^{-1-}$ cultures. We also showed that adult connexin $30^{\mathrm{T} 5 \mathrm{M} / \mathrm{T} 5 \mathrm{M}}$ mice, when probed by auditory brainstem recordings, exhibit a mild but significant increase in their hearing thresholds, of about $15 \mathrm{~dB}$ at all frequencies [28]. By contrast, connexin $30^{-/-}$mice are profoundly deaf $[28,43]$. The present experiments and our previous work confirm cochlear organotypic cultures as an attractive test ground to explore the intricacies of connexin expression regulation and function. In addition, our findings support the notion that connexin $30^{-/}$mice are a model for humans in which large deletions in the DFNB1 locus lead to down-regulation of both GJB6 and GJB2 and to profound deafness [13].

It is well known that electrical conductance and permeability to solutes other than small inorganic ions are not directly related $[8,20,44]$. Even the junctional permeability to fluorescent probes may not be directly related to electrical coupling $[28,45]$. We believe that the methodology described in the present article may help clarifying this complex relationship when used in combination with other complementary techniques, particularly those that (i) provide a direct estimate of the unitary permeability to signaling molecules [7] and (ii) aid data interpretation by the use of Molecular Dynamics [34].

\section{Conclusions}

Here we present a combined electrophysiological and optical approach to visualize rapidly and quantify connectivity in cell networks coupled by gap junctions. Our digital phase-sensitive detector of Vf2.1.Cl fluorescence emission allows greater sensitivity and better time resolution compared to classical tracer-based techniques, and permitted us to track dynamically intercellular connectivity down to the $10^{\text {th }}$ order in non-sensory cell networks of the developing cochlea. Despite the fact that the results shown here were obtained in specific cell models (cochlear non-sensory cells, HeLa cells) we believe that our method is of general interest and can be seamlessly extended to a variety of biological systems, as well as to other connexinrelated disease conditions [10-12].

\section{Methods}

\section{Reagents and drugs}

Vf2.1.Cl [18] was provided by Roger Y. Tsien (University of California, San Diego). Carbenoxolone (CBX), pluronic F-127, Hanks' balanced salt solutions (HBSS) and the salts used to prepare solutions were purchased from Sigma-Aldrich. Lipofectamine, Dulbecco's modified Eagle's medium (DMEM/F12) and fetal bovine serum (FBS) were purchased from Life Technologies. Cell Tak was purchased from Becton Dickinson.

\section{Cochlear organotypic cultures}

Cochleae were dissected from P5 mouse pups in ice-cold Hepes buffered (10 mM, pH 7.2) HBSS, placed onto glass coverslips coated with $185 \mu \mathrm{g} / \mathrm{ml}$ of Cell Tak and incubated overnight at $37^{\circ} \mathrm{C}$ in DMEM/F12 supplemented with FBS 5\%. 


\section{HeLa cells}

A clone of HeLa cells essentially devoid of connexins was provided by Klaus Willecke (University of Bonn, Germany) and cultured according to standard procedures. Twenty four hours after plating, a lipofectamine transfection system was used to transiently transfect these communication-incompetent HeLa cells with hCx26-CFP, a previously described human connexin26 construct tagged with the cyan fluorescent protein (CFP) at its carboxyl terminal end [20].

\section{Electrophysiology and fluorescence imaging}

All experiments were performed at room temperature $\left(22-25^{\circ} \mathrm{C}\right)$. Cochlear or HeLa cell cultures were transferred to the stage of an upright wide-field fluorescence microscope (BX51, Olympus) and continually superfused with EXM, an extracellular medium containing (in $\mathrm{mM}$ ): $\mathrm{NaCl} 138, \mathrm{KCl} 5, \mathrm{CaCl}_{2} 2, \mathrm{NaH}_{2} \mathrm{PO}_{4} 0.3, \mathrm{KH}_{2} \mathrm{PO}_{4}$ 0.4, Hepes-NaOH 10, d-glucose 6 (pH 7.2, 300 mOsm). Glass capillaries for patch clamp recordings were formed on a vertical puller (PP-83, Narishige, Japan) from 1.5-mm outer diameter borosilicate glass (G85150T-4, Warner Instruments) and filled with an intracellular solution containing (in $\mathrm{mM}$ ): $\mathrm{KCl} 134, \mathrm{NaCl} 4, \mathrm{MgCl}_{2} 1$, HEPES 20, EGTA 10 (adjusted to $\mathrm{pH} 7.3$ with $\mathrm{KOH}, 290$ mOsm) and filtered through $0.22 \mu \mathrm{m}$ pores (Millipore). Pipette resistances were 3-4 MOhm when immersed in the EXM bath. For whole-cell (paired) patch clamp recordings, cell 1 was maintained under voltage clamp conditions with a patch clamp amplifier (Model 2400, AM Systems) while cell 2 was kept under current clamp conditions with a second amplifier (EPC-7, HeKa). Current and voltage were filtered at $3 \mathrm{kHz}$ by an 8 pole Bessel filter and sampled at $20 \mathrm{kHz}$ using a standard laboratory interface (Digidata 1440A, Molecular Devices) controlled by the PClamp 10 software (Molecular Devices).

To visualize hCx26-CFP, transfected HeLa cells were illuminated by light from a $385 \mathrm{~nm}$ LED (M385L2, Thorlabs) passing through a D390/70X filter (Chroma) and directed onto the sample through a 440 dclp dichromatic mirror (Chroma) while CFP emission was selected by an ET480/40M filter (Chroma).

For voltage imaging, cochlear or HeLa cell cultures were incubated for $15 \mathrm{~min}$ at $37^{\circ} \mathrm{C}$ in EXM supplemented with Vf2.1.Cl (200 nM) and pluronic F-127 (0.1\% w/v), thereafter cultures were continually superfused with EXM. Vf2.1.Cl fluorescence was excited by light from a $470 \mathrm{~nm}$ LED (M470L2, Thorlabs) passing through a BP460-480 filter (Olympus) and directed onto the sample through a 515 dcxr dichromatic mirror (Chroma) while Vf2.1.Cl fluorescence emission was selected by an ET535/30M filter (Chroma). All fluorescence images were formed by a $60 \times$ water immersion objective (NA 1.0, Fluor, Nikon) and projected on a scientific-grade CCD camera (SensiCam;
PCO AG) controlled by software developed in the laboratory. Image sequences of $\mathrm{Vf} 2.1 . \mathrm{Cl}$ fluorescence were acquired continuously at 10 frames per second with $100 \mathrm{~ms}$ exposure time. To synchronize image acquisition and electrical recordings, we sampled the $5 \mathrm{~V}$ pulse (FVAL) that signals active exposure of the CCD camera [46]. $\mathrm{Vf2.1.Cl}$ signals were measured as relative changes of fluorescence emission intensity $\left(\Delta F / F_{0}\right)$, where $F_{0}$ is prestimulus fluorescence, $F$ is fluorescence at time $t$ and $\Delta F=F-F_{0}$.

Miller et al. reported that Vf2.1.Cl and other PeTbased voltage indicators have a slower rate of bleaching and are less toxic than the FRET-based dyes [18]. We did not make a direct comparison between these two classes of indicators. However, in our hands patch clamp recordings from cochlear non-sensory cells in Vf2.1.Cl loaded cultures were stable for tens of minutes during continuous illumination with the LED used to excite dye's fluorescence. In addition, we did not notice any visible sign of cellular degeneration.

\section{Image processing}

Vf2.1.Cl fluorescence image sequences were stored on disk and processed off-line using the Matlab R2011a software package (The MathWorks, Inc.) as described hereafter. Following electrical stimulation of cell 1 with a carrier wave at frequency $v$, each image was preprocessed by applying a 3-by-3 mean spatial filter to reduce acquisition noise. To correct for photobleaching, we first estimated its time course by performing a low order polynomial fit to the $\left(\Delta F / F_{0}\right)(t ; x, y)$ data at each pixel location $(x, y)$; the fitting function $P(t ; x, y)$ was then subtracted from the $\left(\Delta F / F_{0}\right)(t ; x, y)$ signal, yielding an effectively high-pass filtered trace

$$
f(t ; x, y)=\frac{\Delta F(t ; x, y)}{F_{0}(x, y)}-P(t ; x, y)
$$

The purpose of using a phase-sensitive detector is to extract the signal amplitude $A(x, y)$ from the preprocessed single pixel signal

$$
f(t ; x, y)=A(x, y) \cos (2 \pi v t-\theta)
$$

where $\theta$ is a constant phase delay [27]. We performed the extraction procedure in two steps:

Step 1: demodulation. $f(t ; x, y)$ was multiplied by two orthogonal reference signals

$$
\begin{aligned}
& V_{1}^{r e f}(t)=\cos (2 \pi \nu t) \\
& V_{2}^{r e f}(t)=\sin (2 \pi v t)
\end{aligned}
$$


(see Figure 2B) yielding two output signals of the form

$$
\begin{aligned}
& f_{1}(t ; x, y)=V_{1}^{r e f}(t) \cdot f(t ; x, y)=A(x, y) \cos (2 \pi v t-\theta) \cos (2 \pi v t) \\
& f_{2}(t ; x, y)=V_{2}^{r e f}(t) \cdot f(t ; x, y)=A(x, y) \cos (2 \pi v t-\theta) \sin (2 \pi v t)
\end{aligned}
$$

Considering the trigonometric identities

$$
\begin{aligned}
& \cos (a) \cos (b)=\frac{1}{2}[\cos (a-b)+\cos (a+b)] \\
& \cos (a) \sin (b)=\frac{1}{2}[\sin (a+b)-\sin (a-b)]
\end{aligned}
$$

the two output signals can be written as

$$
\begin{aligned}
& f_{1}(t ; x, y)=\frac{1}{2} A(x, y)[\cos (\theta)+\cos (2 \pi(2 v) t-\theta)] \\
& f_{2}(t ; x, y)=\frac{1}{2} A(x, y)[\sin (2 \pi(2 v) t-\theta)+\sin (\theta)]
\end{aligned}
$$

and are seen to consist of a DC signal proportional to the amplitude $A(x, y)$ of the original function $f(t ; x, y)$ and a time-dependent component with frequency $2 v$.

Step 2: Amplitude estimation. The time-dependent component was filtered out by time integration of $f_{1}(t ; x, y)$ and $f_{2}(t ; x, y)$. In the absence of noise, integration over a single carrier wave cycle would yield the DC components of $f_{1}(t ; x, y)$ and $f_{2}(t ; x, y)$ :

$$
\begin{aligned}
& a_{1}(x, y)=\frac{1}{2} A(x, y) \cos (\theta) \\
& a_{2}(x, y)=\frac{1}{2} A(x, y) \sin (\theta)
\end{aligned}
$$

In practice, integration is better performed over a number $N$ of cycles to reduce contributions from various noise sources (see Figure 2E). Finally, the amplitude of $f(t ; x, y)$ was computed as

$$
A(x, y)=2 \sqrt{\left[a_{1}(x, y)\right]^{2}+\left[a_{2}(x, y)\right]^{2}}
$$

The reference level $A(x, y)=0$ was set by applying the above algorithm to the pre-stimulus trace (i.e. to the trace segment that preceded cell 1 stimulation by the carrier wave).

\section{Statistical analysis}

Means are quoted \pm standard error of the mean (s.e.m.) and $\mathrm{p}$-values are indicated by letter $p$. Statistical comparisons were made using the Mann-Whitney $U$ test [47] and $p<0.05$ was selected as the criterion for statistical significance.

\section{Animal handling}

Animal handling was approved by the Ethical Committee of Padua University (Comitato Etico di Ateneo per la Sperimentazione Animale, C.E.A.S.A.) project n. 54/2009, protocol n. 51731.

\section{Additional files}

Additional file 1: Movie S1. Effect of carrier wave stimulation. This video shows raw fluorescence signals from a P5 wild type cochlear organotypic culture loaded with Vf2.1.Cl; a patch pipette entering from the left delivers a sinusoidal voltage stimulation at $0.5 \mathrm{~Hz}$ (carrier wave) to a cell maintained under whole-cell conditions.

Additional file 2: Movie S2. Effect of $\mathrm{CO}_{2}$ application. This is the video sequence from which frames in Figure 5 were extracted. It shows processed fluorescence signals from a cochlear organotypic culture loaded with Vf2.1.Cl during application of $\mathrm{CO}_{2}$ (see main text for details).

Additional file 3: Movie S3. Lucifer Yellow delivery via patch pipette to a non-sensory cell of the lesser epithelial ridge. This video sequence was captured from a P5 wild type cochlear organotypic culture while delivering Lucifer Yellow dissolved at a concentration of $225 \mu \mathrm{M}$ in the intracellular solution described in the Methods. Note that the patch pipette used for dye microinjection had the same physical characteristics (mouth diameter, electrical resistance) of those utilized for the delivery of the carrier wave signals of our digital phase-sensitive detector.

\section{Abbreviations}

VF: VoltageFluor; GJB2: Gene encoding gap junction beta-2 protein; GJB6: Gene encoding gap junction beta-6 protein; CBX: Carbenoxolone; CFP: Cyan fluorescent protein; FRAP: Fluorescence recovery after photobleaching; ROI(s): Region(s) of interest.

\section{Competing interests}

The authors declare that they have no competing interests.

\section{Authors' contributions}

FM and FC respectively designed and performed the experiments; FC analyzed data; FM wrote the paper. Both authors read and approved the final manuscript.

\section{Acknowledgements}

Supported by Telethon Italy grant GGP13114, MIUR PRIN grant $n$. 2009CCZSES and Fondazione CARIPARO 2010 Ph.D. fellowship grant No. PARO103433 to FM. We thank R.Y. Tsien for the generous gift of $V f 2.1 . C l$.

\section{Author details}

1Dipartimento di Fisica e Astronomia "G. Galilei", Università di Padova, Padova 35131, Italy. ${ }^{2}$ Istituto Veneto di Medicina Molecolare, Fondazione per la Ricerca Biomedica Avanzata, Via G. Orus, 2, Padova 35129, Italy. ${ }^{3}$ Istituto di Neuroscienze, Consiglio Nazionale delle Ricerche, Padova 35131, Italy.

Received: 30 August 2013 Accepted: 1 October 2013

Published: 21 October 2013

\section{References}

1. Goodenough DA, Paul DL: Gap junctions. Cold Spring Harb Perspect Biol 2009, 1:a002576.

2. Bennett MV, Zukin RS: Electrical coupling and neuronal synchronization in the Mammalian brain. Neuron 2004, 41:495-511.

3. McCracken CB, Roberts DC: Neuronal gap junctions: expression, function, and implications for behavior. Int Rev Neurobiol 2006, 73:125-151.

4. Yu YC, He S, Chen S, Fu Y, Brown KN, Yao XH, Ma J, Gao KP, Sosinsky GE, Huang K, Shi SH: Preferential electrical coupling regulates neocortical lineage-dependent microcircuit assembly. Nat 2012, 486:113-117.

5. Kirchhoff S, Nelles E, Hagendorff A, Kruger O, Traub O, Willecke K: Reduced cardiac conduction velocity and predisposition to arrhythmias in connexin40-deficient mice. Curr Biol 1998, 8:299-302.

6. Jansen JA, van Veen TA, de Bakker JM, van Rijen HV: Cardiac connexins and impulse propagation. J Mol Cell Cardiol 2010, 48:76-82.

7. Hernandez VH, Bortolozzi M, Pertegato V, Beltramello M, Giarin M, Zaccolo M, Pantano S, Mammano F: Unitary permeability of gap junction channels to second messengers measured by FRET microscopy. Nat Methods 2007, 4:353-358.

8. Harris AL: Connexin channel permeability to cytoplasmic molecules. Prog Biophys Mol Biol 2007, 94:120-143.

9. Wallraff A, Kohling R, Heinemann U, Theis M, Willecke K, Steinhauser C: The impact of astrocytic gap junctional coupling on potassium buffering in the hippocampus. J Neurosci 2006, 26:5438-5447.

10. Wei CJ, Xu X, Lo CW: Connexins and cell signaling in development and disease. Annu Rev Cell Dev Biol 2004, 20:811-838. 
11. Laird DW: Life cycle of connexins in health and disease. Biochem J 2006, 394:527-543.

12. Dobrowolski R, Willecke $\mathrm{K}$ : Connexin-caused genetic diseases and corresponding mouse models. Antioxid Redox Signal 2009, 11:283-295.

13. del Castillo FJ, del Castillo I: The DFNB1 subtype of autosomal recessive non-syndromic hearing impairment. Front Biosci 2011, 16:3252-3274.

14. Lautermann J, ten Cate WJ, Altenhoff P, Grummer R, Traub O, Frank H, Jahnke K, Winterhager E: Expression of the gap-junction connexins 26 and 30 in the rat cochlea. Cell Tissue Res 1998, 294:415-420.

15. Lautermann J, Frank HG, Jahnke K, Traub O, Winterhager E: Developmental expression patterns of connexin 26 and -30 in the rat cochlea. Dev Genet 1999, 25:306-311.

16. Cohen-Salmon M, del Castillo FJ, Petit C: Connexins Responsiblbe for Hereditary Deafness - The Tale Unfolds. In Gap Junctions in Development and Disease. Edited by Winterhager E. Berlin: Springer-Verlag; 2005:111-134.

17. Hou M, Li Y, Paul DL: A novel, highly sensitive method for assessing gap junctional coupling. J Neurosci Methods 2013, 220(1):18-23.

18. Miller EW, Lin JY, Frady EP, Steinbach PA, Kristan WB Jr, Tsien RY: Optically monitoring voltage in neurons by photo-induced electron transfer through molecular wires. Proc Natl Acad Sci USA 2012, 109:2114-2119.

19. Gale JE, Piazza V, Ciubotaru CD, Mammano F: A mechanism for sensing noise damage in the inner ear. Curr Biol 2004, 14:526-529.

20. Beltramello M, Piazza V, Bukauskas FF, Pozzan T, Mammano F: Impaired permeability to $\operatorname{Ins}(1,4,5) \mathrm{P}_{3}$ in a mutant connexin underlies recessive hereditary deafness. Nat Cell Biol 2005, 7:63-69.

21. Piazza V, Ciubotaru CD, Gale JE, Mammano F: Purinergic signalling and intercellular $\mathrm{Ca}^{2+}$ wave propagation in the organ of Corti. Cell Calcium 2007, 41:77-86.

22. Anselmi F, Hernandez VH, Crispino G, Seydel A, Ortolano S, Roper SD, Kessaris N, Richardson W, Rickheit G, Filippov MA, et al: ATP release through connexin hemichannels and gap junction transfer of second messengers propagate $\mathrm{Ca}^{2+}$ signals across the inner ear. Proc Natl Acad Sci USA 2008, 105:18770-18775.

23. Majumder P, Crispino G, Rodriguez L, Ciubotaru CD, Anselmi F, Piazza V, Bortolozzi M, Mammano F: ATP-mediated cell-cell signaling in the organ of Corti: the role of connexin channels. Purinergic Signal 2010, 6:167-187.

24. Mammano F: ATP-dependent intercellular $\mathrm{Ca}^{2+}$ signaling in the developing cochlea: Facts, fantasies and perspectives. Semin Cell Dev Biol 2013, 24:31-39.

25. Verselis VK, Srinivas M: Connexin channel modulators and their mechanisms of action. Neuropharmacology 2013. Available online 15 April 2013, In Press, http://dx.doi.org/10.1016/j.neuropharm.2013.03.020.

26. Davies R, Graham J, Canepari M: Light sources and cameras for standard in vitro membrane potential and high-speed ion imaging. J Microsc 2013, 251:5-13.

27. Cova S, Longoni A, Freitas I: Versatile digital lock-in detection technique: application to spectrofluorometry and other fields. Rev Sci Instrum 1979, 50:296.

28. Schutz M, Scimemi $P$, Majumder $P$, De Siati RD, Crispino G, Rodriguez $L$, Bortolozzi M, Santarelli R, Seydel A, Sonntag S, et al: The human deafnessassociated connexin 30 T5M mutation causes mild hearing loss and reduces biochemical coupling among cochlear non-sensory cells in knock-in mice. Hum Mol Genet 2010, 19:4759-4773.

29. Lagostena L, Cicuttin A, Inda J, Kachar B, Mammano F: Frequency dependence of electrical coupling in Deiters' cells of the guinea pig cochlea. Cell Commun Adhes 2001, 8:393-399.

30. Bukauskas FF, Verselis VK: Gap junction channel gating. Biochim Biophys Acta 2004, 1662:42-60.

31. Beltramello M, Bicego M, Piazza V, Ciubotaru CD, Mammano F, D'Andrea P: Permeability and gating properties of human connexins 26 and 30 expressed in HeLa cells. Biochem Biophys Res Commun 2003, 305:1024-1033.

32. Bicego M, Beltramello M, Melchionda S, Carella M, Piazza V, Zelante L, Bukauskas FF, Arslan E, Cama E, Pantano S, et al: Pathogenetic role of the deafness-related M34T mutation of Cx26. Hum Mol Genet 2006, 15:2569-2587.

33. Jin L, Han Z, Platisa J, Wooltorton JR, Cohen LB, Pieribone VA: Single action potentials and subthreshold electrical events imaged in neurons with a fluorescent protein voltage probe. Neuron 2012, 75:779-785

34. Zonta F, Polles G, Sanasi MF, Bortolozzi M, Mammano F: The 3.5 angstrom $\mathrm{X}$-ray structure of the human connexin 26 gap junction channel is unlikely that of a fully open channel. Cell Commun Signal 2013, 11:15.
35. Wade MH, Trosko JE, Schindler M: A fluorescence photobleaching assay of gap junction-mediated communication between human cells. Sci 1986, 232:525-528.

36. Ortolano S, Di Pasquale G, Crispino G, Anselmi F, Mammano F, Chiorini JA: Coordinated control of connexin 26 and connexin 30 at the regulatory and functional level in the inner ear. Proc Natl Acad Sci USA 2008, 105:18776-18781.

37. Yum SW, Zhang J, Valiunas V, Kanaporis G, Brink PR, White TW, Scherer SS: Human connexin26 and connexin30 form functional heteromeric and heterotypic channels. Am J Physiol Cell Physiol 2007, 293:C1032-1048.

38. Santos-Sacchi J: Isolated supporting cells from the organ of Corti: some whole cell electrical characteristics and estimates of gap junctional conductance. Hear Res 1991, 52:89-98.

39. Forge A, Becker D, Casalotti S, Edwards J, Marziano N, Nevill G: Gap junctions in the inner ear: comparison of distribution patterns in different vertebrates and assessement of connexin composition in mammals. J Comp Neurol 2003, 467:207-231.

40. Bukauskas FF, Jordan K, Bukauskiene A, Bennett MV, Lampe PD, Laird DW, Verselis VK: Clustering of connexin 43-enhanced green fluorescent protein gap junction channels and functional coupling in living cells. Proc Natl Acad Sci USA 2000, 97:2556-2561.

41. Palacios-Prado N, Sonntag S, Skeberdis VA, Willecke K, Bukauskas FF: Gating, permselectivity and $\mathrm{pH}$-dependent modulation of channels formed by connexin57, a major connexin of horizontal cells in the mouse retina. J Physiol 2009, 587:3251-3269.

42. Palacios-Prado N, Briggs SW, Skeberdis VA, Pranevicius M, Bennett MV, Bukauskas FF: pH-dependent modulation of voltage gating in connexin45 homotypic and connexin45/connexin43 heterotypic gap junctions. Proc Natl Acad Sci USA 2010, 107:9897-9902.

43. Teubner B, Michel V, Pesch J, Lautermann J, Cohen-Salmon M, Sohl G, Jahnke K, Winterhager E, Herberhold C, Hardelin JP, et al: Connexin30 (Gjb6)-deficiency causes severe hearing impairment and lack of endocochlear potential. Hum Mol Genet 2003, 12:13-21.

44. Goldberg GS, Lampe PD, Nicholson BJ: Selective transfer of endogenous metabolites through gap junctions composed of different connexins. Nat Cell Biol 1999, 1:457-459.

45. Ek-Vitorin JF, King TJ, Heyman NS, Lampe PD, Burt JM: Selectivity of connexin 43 channels is regulated through protein kinase C-dependent phosphorylation. Circ Res 2006, 98:1498-1505.

46. Mammano F, Canepari M, Capello G, ljaduola RB, Cunei A, Ying L, Fratnik F, Colavita A: An optical recording system based on a fast CCD sensor for biological imaging. Cell Calcium 1999, 25:115-123.

47. Mann HB, Whitney DR: On a Test of Whether one of Two Random Variables is Stochastically Larger than the Other. Ann Math Stat 1947, 18:50-60.

\section{doi:10.1186/1478-811X-11-78}

Cite this article as: Ceriani and Mammano: A rapid and sensitive assay of intercellular coupling by voltage imaging of gap junction networks. Cell Communication and Signaling 2013 11:78.

\section{Submit your next manuscript to BioMed Central and take full advantage of:}

- Convenient online submission

- Thorough peer review

- No space constraints or color figure charges

- Immediate publication on acceptance

- Inclusion in PubMed, CAS, Scopus and Google Scholar

- Research which is freely available for redistribution 